\title{
FingerTac - A Wearable Tactile Thimble for Mobile Haptic Augmented Reality Applications ${ }^{\star}$
}

\author{
Thomas Hulin ${ }^{1,2}$, Michael Rothammer ${ }^{1}$, Isabel Tannert ${ }^{1}$, Suraj Subramanyam \\ Giri $^{1}$, Benedikt Pleintinger ${ }^{1}$, Harsimran Singh ${ }^{1,2}$, Bernhard Weber ${ }^{1}$, and \\ Christian $\mathrm{Ott}^{1,2}$ \\ 1 Institute of Robotics and Mechatronics, German Aerospace Center (DLR), \\ D-82234 Wessling, Germany \\ 2 Centre for Tactile Internet with Human-in-the-Loop (CeTI), \\ Cluster of Excellence at TU Dresden, Germany
}

\begin{abstract}
FingerTac is a novel concept for a wearable augmented haptic thimble. It makes use of the limited spatial discrimination capabilities of vibrotactile stimuli at the skin and generates tactile feedback perceived at the bottom center of a fingertip by applying simultaneous vibrations at both sides of the finger. Since the bottom of the finger is thus kept free of obstruction, the device is well promising for augmented haptic applications, where real world interactions need to be enriched or amalgamated with virtual tactile feedback. To minimize its lateral dimension, the vibration actuators are placed on top of the device, and mechanical links transmit the vibrations to the skin. Two evaluation studies with $\mathrm{N}=10$ participants investigate (i) the loss of vibration intensity through these mechanical links, and (ii) the effect of lateral displacement between stimulus and induced vibration. The results of both studies support the introduced concept of the FingerTac.
\end{abstract}

Keywords: Augmented Haptics · Tactile Feedback · Wearable Devices

\section{Introduction}

Compared to pure cutaneous haptic feedback on the skin, kinesthetic feedback provides forces and torques. Such feedback is essential for delicate tasks that occur e.g. in telerobotic applications, where a user is required to intuitively control the forces applied by the remote robot. However, this benefit comes at the price of complex mechanical hardware systems. While a couple of welldeveloped kinesthetic devices for force and torque feedback for the hand exist, the situation is different for fingers. Hand exoskeletons are often obstructive and have limited force capabilities. In comparison, tactile feedback can be realized by much leaner and lighter devices.

\footnotetext{
* Funded by the German Research Foundation (DFG, Deutsche Forschungsgemeinschaft) as part of Germany's Excellence Strategy - EXC 2050/1 - Project ID 390696704 - Cluster of Excellence "Centre for Tactile Internet with Humanin-the-Loop" (CeTI) of Technische Universität Dresden.
} 
A lot of research was conducted towards incorporating several types of strategies into delivering tactile feedback to the user. Pin-arrays use dense arrangements of skin contactors that exert pressure onto the skin to display tactile information. Such pin-arrays can be actuated using electromagnets [28], piezoelectric crystals [27], shape memory alloys [26] and pneumatic systems [13]. They can be used to provide braille dot patterns and virtual surface texture, among others, to the user. Although such devices are very effective in conducting tactile information via spatially sampled approximation, they are not mobile or wearable since they usually need a significantly large separate actuation system. Some tactile displays use the principles of electrovibration and electrical stimuli to provide vibrational friction, pressure and vibration at the same time [9].

Certain tactile devices apply multi-DoF force vectors on the user's fingerpad at one or multi contact points. Minamizawa et al. [12] proposed a wearable device comprised of two motors and a movable belt in contact with the user's finger, designed for generating normal and shear stress to deform the fingerpads which simulates a weight sensation of a virtual object. The "hRing" proposed by Pacchierotti et al. [16], is another 2-DoF cutaneous device based on the same principle as [12], and is used to provide normal and tangential stimulus to the proximal phalanx of the index finger. Schorr and Okamura [21] presented an approach that uses a pair of finger mounted devices that deforms the fingerpads to convey interaction information such as grasping, squeezing, pressing, lifting, and stroking. Prattichizzo et al. [17] developed a miniature device with parallel mechanism that applies 3-DoF force onto the fingerpad through a rigid platform which is actuated using cables and miniaturized motors. Leonardis et al. [10], developed a wearable haptic device modulates the contact forces, in 3-DoF, by stretching the skin. Solazzi et al. [23] designed a portable interface with fast transient that could display contact and orientation information to the user. The device was later upgraded by adding a voice coil, enabling it to simultaneously deliver the virtual surface's orientation and a wide frequency bandwidth of tactile stimulation [5]. A ferro-fluid based tactile device was fabricated by Singh et al. [22], that can also transmit both orientation and texture information at the same time. Although such devices are wearable, the overall structure is not compact enough to be worn on more than a couple of fingers as it obstructs the user's natural workspace.

By far, vibrational feedback has outnumbered other forms of tactile feedback due to its advantages of being compact, lightweight, wearable and portable. Voice coil motors and piezoelectric materials are some of the common actuators used to generate non-directional feedback, such as vibration patterns, for wearables [6], VR [15] or video games. They can also be used to transmit directional cues through a wearable belt [25], vest [3] of wristband [18], or for communicating emotions [1]. Some researchers also use other actuation technologies to generate vibrotactile feedback, for instance shape memory alloys [20] or electro-active polymers $[2,14]$.

While most of the above mentioned devices only work with virtual reality, there are others that can be used for augmented reality as well, as these de- 


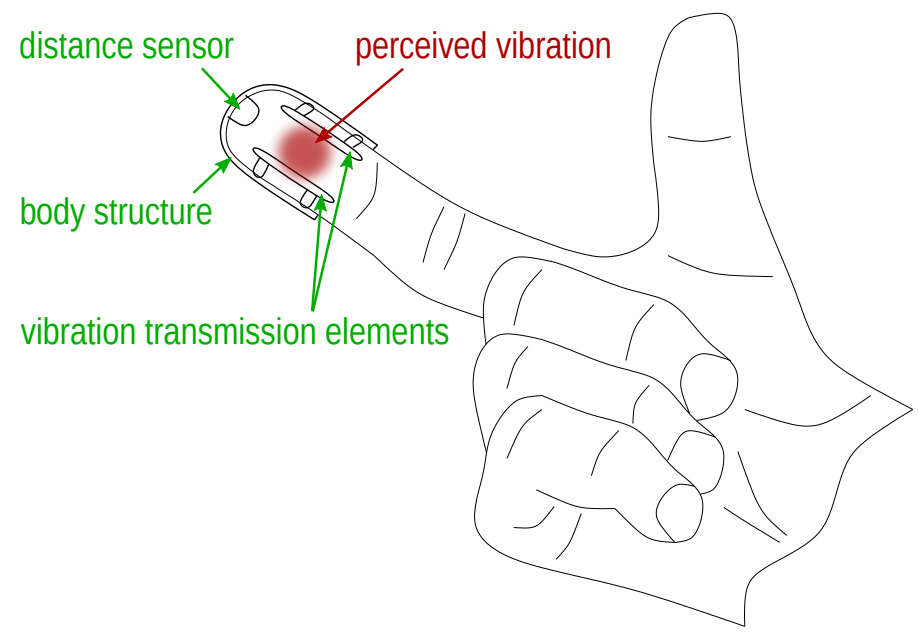

Fig. 1. Conceptual sketch of the FingerTac.

vices interfere only minimally when interacting with real objects. Fani et al. [4] presented the Wearable-Fabric Yielding Display which can convey softness information for passive and active haptic exploration. It was also shown that by controlling the volumetric distribution of acoustic field through a two-dimensional phased ultrasound array, volumetric haptic shapes can be rendered in mid-air [11, 7]. One of its shortcomings is the limited workspace, where the ultrasound arrays must create a shape within the functional volume of the device, and is therefore not wearable. Taking the research forward in the direction of wearablity, Spelmezan et al. [24] showed that by placing an array of ultrasound emitters on the back of the hand and timing the pulses, a tactile sensation on the palm due to constructive interference can be created. However, ultrasonic emitters are not small enough to make such a technology wearable at the moment.

This paper follows a different approach and introduces FingerTac, a novel concept for a tactile thimble. While various approaches exist to generate tactile feedback at a fingertip, the unique feature of our concept is that it generates tactile feedback at the bottom side of a fingertip and at the same time keeps this bottom side unobstructed. This is achieved by inducing vibrational feedback at both sides of a finger and making use of the limited spatial discrimination capabilities of vibrotactile stimuli at the skin. Thus, our device generates perceived vibration in-between the two contact areas where the stimuli are applied. This unique property makes the device well suited for augmented haptic applications, where both real and virtual objects need to be touched and manipulated.

The remainder of this document discusses the conceptual idea of the FingerTac in more detail, describes two demonstrators, and presents evaluation studies as proof of concept. 


\section{Concept and Functional Demonstrators}

The conceptual sketch of the FingerTac is illustrated in Fig. 1. The device induces vibrational feedback via two contact areas that are located on both sides of a fingertip. Vibrational stimuli are applied simultaneously on both contact areas at similar frequencies, such that the perceived vibration lies in-between the two areas at the fingertip's bottom side. At the same time, this central fingertip area is kept uncovered to enable unobstructed interactions with real objects.

To minimize the lateral dimensions of the device, the actuators are located on its top side above the fingernail, and the vibrations are transferred from the actuators to the skin via two vibration transmission elements. Vibrations between these elements and the body structure are mechanically decoupled through a flexible vibration isolation structure. The body structure may also hold different sensors. In particular, a distance sensor at the front of the fingertip would allow for measuring and feeding back the distance of surrounding objects and could thereby extend the possible fields of application of such device. For instance, visually impaired people could benefit from this additional information, which has recently been shown in a pilot study with a vibrotactile wristband [19].

The following sections discuss design criteria that were considered for the FingerTac, and describe the functional demonstrators that were built and used for the conducted user studies.

\subsection{Design Considerations}

Several design criteria are of importance for the FingerTac in order to achieve clear tactile feedback, high wearing comfort, and unhindered natural interaction with the environment. While a wide range of objectives for designing tactile devices are known [8], the following aspects are of particular relevance for the FingerTac due to its nature as wearable augmented haptic device:

Unobtrusive shape: A wearable device needs to ensure kinematic compatibility with the human body movements. Compared to classical tactile devices that are made for interaction in virtual environments, this objective is of high importance for the FingerTac, as the device should be used while interacting with both virtual and real environments. The device should minimally interfere with the fingerpad, so that the user can grasp or interact with real objects.

Low inertia: Minimizing the overall mass of the device is one of the most important design goals for a wearable haptic interaction device. Apart from the overall mass, the distribution of masses also plays an important role especially during rotational movements of the hand. Components with a higher mass should be placed as close as possible to the pivot point or center of the fingers. Heavier components such as the battery can be separated from the device and placed on rather stronger body parts, i.e. on the hand instead of the finger. 


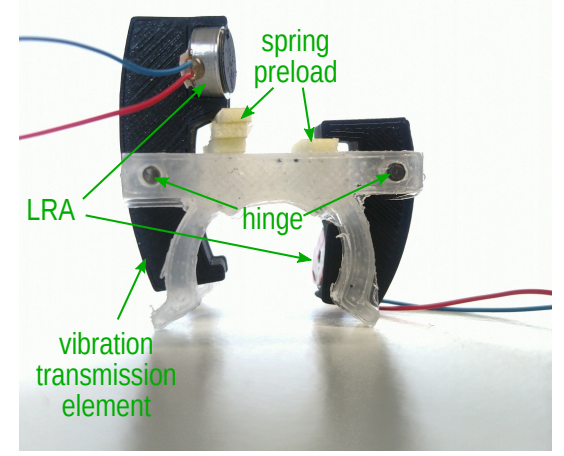

(a) evaluation prototype

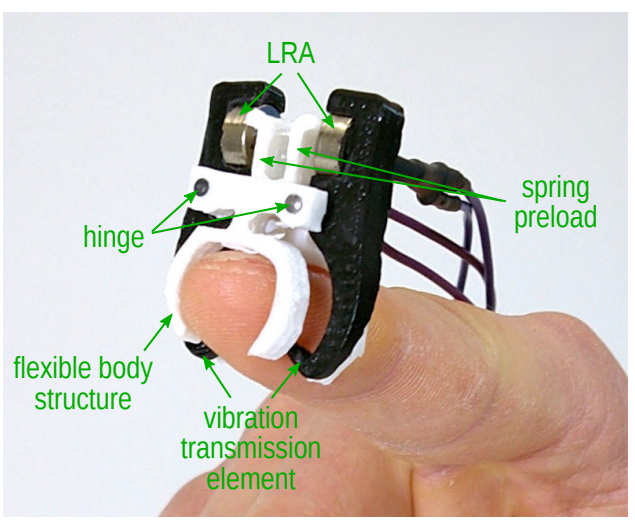

(b) functional demonstrator

Fig. 2. Evaluation prototype and functional demonstrator to evaluate the FingerTac concept.

High comfort: A wearable haptic device should be comfortable and easily adaptable to the user's finger. High contact pressure and sharp edges should be avoided and a wide range of finger sizes should be covered.

These objectives highly influenced the design of the functional demonstrators of the FingerTac as described below in the next section.

\section{$2.2 \quad$ Functional Demonstrators}

Fig. 2 shows two different devices that were developed to evaluate the principal idea of our concept and to compare how much signal strength is lost if vibration is transmitted via vibration transmission elements. The evaluation prototype of Fig. 2(a) was developed first to evaluate the effect of the transmission elements compared to direct contact between actuator and skin. It has two different transmission elements, with the actuator placed on the top and bottom side respectively.

The functional demonstrator shown in Fig. 2(b) has two identical vibration transmission elements. They allow for placing the vibrotactile actuators on the top side of the device (i.e. on the fingernail side). Hence, they make it possible to reduce the device's dimensions on the bottom and the sides of a finger. While the vibration transmission elements are $3 \mathrm{~d}$-printed out of traditional PLA (polylactic acid), a flexible material is used for the body structure such that the device can be used on fingers of different dimensions. The vibration actuators are linear resonant actuators (LRA) that provide high oscillation amplitudes and fast response dynamics. Their nominal operating frequency is $235 \mathrm{~Hz}$ and hence in the range in which the skin has high sensitivity. The technical system specifications of the built demonstrator are summarized in Table 1. 
Table 1. System specifications of the FingerTac.

\begin{tabular}{ll}
\hline \multicolumn{2}{c}{ system specifications } \\
\hline size & $16 \mathrm{~mm} \times 24 \mathrm{~mm} \times 31 \mathrm{~mm}(\mathrm{l} \times \mathrm{w} \times \mathrm{h})$ \\
weight & approx. $6 \mathrm{~g}(+$ cables $3 \mathrm{~g})$ \\
supported diam. range & $11 \mathrm{~mm}-18 \mathrm{~mm}$ \\
actuators & $2 \mathrm{LRAs}, \varnothing 8 \mathrm{~mm} \times 3.2 \mathrm{~mm}, 2 \mathrm{~V}$ \\
rated frequency & $235 \mathrm{~Hz}$ \\
microcontroller & ESP32, Espressif Systems, $2.4 \mathrm{GHz}$ \\
\hline
\end{tabular}

\section{Evaluation Studies}

In order to evaluate the suitability of the concept and to determine the reduction of tactile stimulus due to the transmission of vibrations through the vibration transmission elements, studies were conducted on the evaluation prototype and the functional demonstrator with $\mathrm{N}=10$ subjects each.

\subsection{Study on the Evaluation Prototype}

The first study investigates the influence of the vibration transmission element. The evaluation prototype of Fig. 2(a) was used for this purpose.

Sample: Nine right-handed and one left-handed employees and students (9 males and 1 female) from DLR took part in this study (age $=26.3 \pm 2.83$ years, ranging from 23-32). Four of these participants had previous experience with haptic feedback systems.

Experimental Task and Design: The device was put on the index finger of the participant. Rectangular vibration patterns were activated subsequently in random order on each actuator (see Fig. 3). Three different time periods for the patterns were used $\left(t_{\text {on }}=50 \mathrm{~ms}, t_{\text {off }}=\{20,100,200\} \mathrm{ms}\right)$. After each pattern, the participant had to repeat the experiment with changed direction of the device, i.e. the device was rotated by $180^{\circ}$ such that the short and long transmission elements exchanged their positions.

Procedure: After a short introduction and signing informed consent, the evaluation prototype was put on the index finger of the participant's dominant hand. After the experiment, each participant was asked to fill out a questionnaire.

Results: The participants rated the experiments on a 7-point Likert-type scale ("Which actuator has the better localizable vibration?", 1=direct contact with skin; $7=$ vibration transmission element, and "How is the intensity of vibration?", 1=very low; $7=$ very high). The vibration transmission element resulted in a better score for localized vibration $(\mathrm{M}=4.75, \mathrm{SD}=1.7)$. The obvious reason for the higher localization ratings for the vibration transmission element is because the vibration is applied over a smaller skin area compared to the actuator that is in direct contact with the skin.

For the intensity, both actuators obtained similar scores $(\mathrm{M}=4.7, \mathrm{SD}=0.9$ for the vibration transmission element vs. $\mathrm{M}=4.4, \mathrm{SD}=1.2$ for direct contact). These 


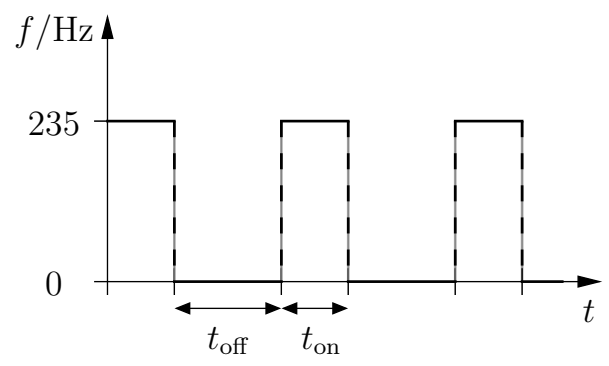

Fig. 3. Vibration pattern used in the studies. The nominal vibration frequency for the actuators was $235 \mathrm{~Hz}$.

results indicate that the vibration transmission element is a suitable alternative to actuators in direct contact with the skin.

\subsection{Study on the Functional Demonstrator}

The goal of the second study was to show that by providing stimulus to the sides of a fingertip, the vibrations can be felt in-between at the fingerpad, and that participants are able to discriminate between different vibration patterns.

Sample: Ten participants ( 8 males and 2 females, 9 right-handers and 1 lefthander) were recruited from the student and staff population of the DLR (age $=25.6 \pm 7.15$ years, ranging from 18-44). All participants read and signed a consent form.

Experimental Task and Design: The participants executed two different tasks.

Task 1 - localization of vibrations: During the first task, the participants were asked to touch an external actuator in such a way that the actuator was located directly between the two vibration contact areas of the FingerTac (see Fig. 4). The actuator was placed on a scale. Participants were advised to keep the weight on the scale between 400 to $600 \mathrm{~g}$, only with their index finger. In this range the vibration intensity of the external actuator is comparable to that of the demonstrator. To improve the participants' mobility and their view on the scale, the cable mounting direction of the FingerTac was modified compared to the original design of Fig. 2(b). In a random order, ten vibrations were presented, some coming from the external actuator, some from the FingerTac itself. After each vibration, the participant had to indicate the origin of the vibration.

Task 2 - discrimination of frequencies: The second task investigated the discrimination capability with regard to different vibration patterns (see Fig. 5). It consisted of a familiarization period and two subtasks. First, four bricks with different colors were placed in front of the participants. Each color represented a different frequency of vibrations (red: $13.33 \mathrm{~Hz}$, yellow: $8.00 \mathrm{~Hz}$, blue: $3.63 \mathrm{~Hz}$ and green: $1.38 \mathrm{~Hz}$ ). These frequencies were implemented as vibration patterns in the form of a rectangular function with fixed activation periods of $t_{\mathrm{on}}=50 \mathrm{~ms}$ 


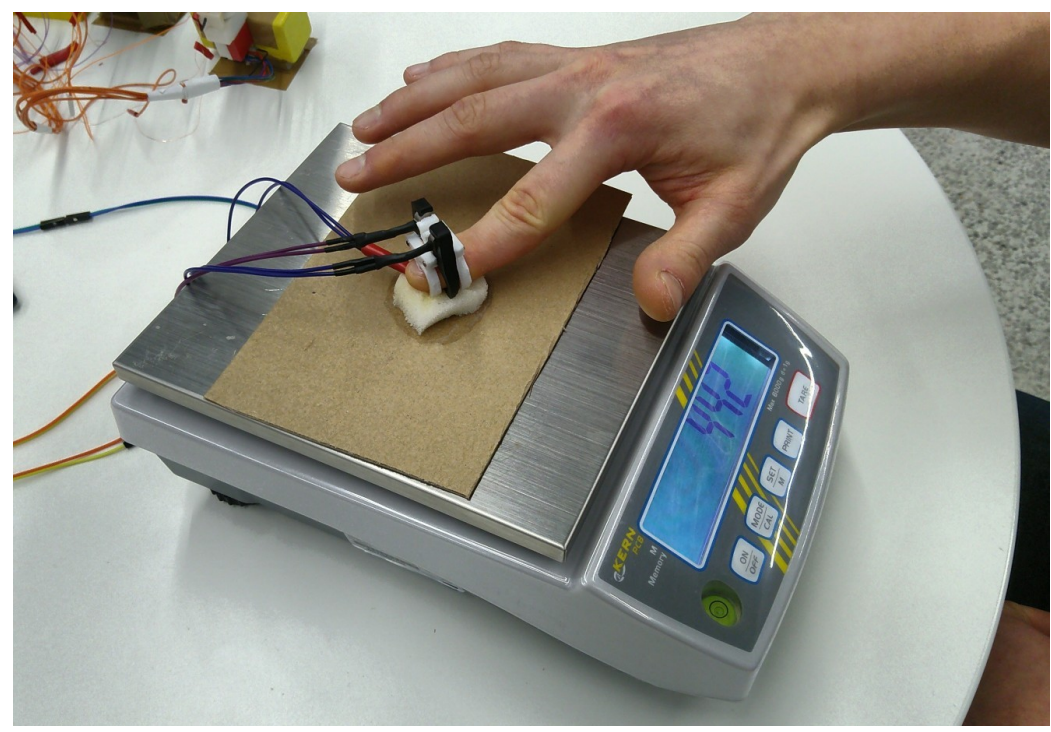

(a) Evaluation setup for the $1^{\text {st }}$ task
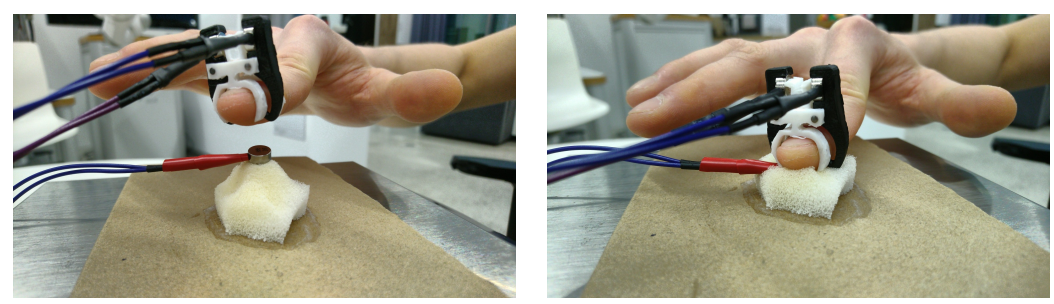

(b) Close-up view on the index finger before and while pressing on the external actuator

Fig. 4. $1^{\text {st }}$ task: localization of vibrations. The participants wore the FingerTac on the index finger of their dominant hand and pressed against an external vibration actuator with a force between 3.92 and $5.89 \mathrm{~N}$ (resp. 400 to $600 \mathrm{~g}$ ).

and different pause times of $t_{\text {off }}=\{25,75,255,675\} \mathrm{ms}$ (see Fig. 3). During the training, participants touched and held the bricks and feeling the different vibration patterns. They were asked to touch each brick at least twice to assure that they are able to recall the patterns during the experiment. As soon as they felt ready, participants were blindfolded.

Frequency detection subtask 1: Next, all bricks were placed in front of them and they were asked to identify a specific color, just by feeling the vibration pattern while grasping and touching the brick. After selecting one, the participants were asked to rate the difficulty of identifying the brick on a 7-point Likert-type scale ("Please rate the difficulty of identifying the color", $1=$ very low; $7=$ very hard). This was repeated six times with a systematic variation of the colors; each color appeared at least once. 


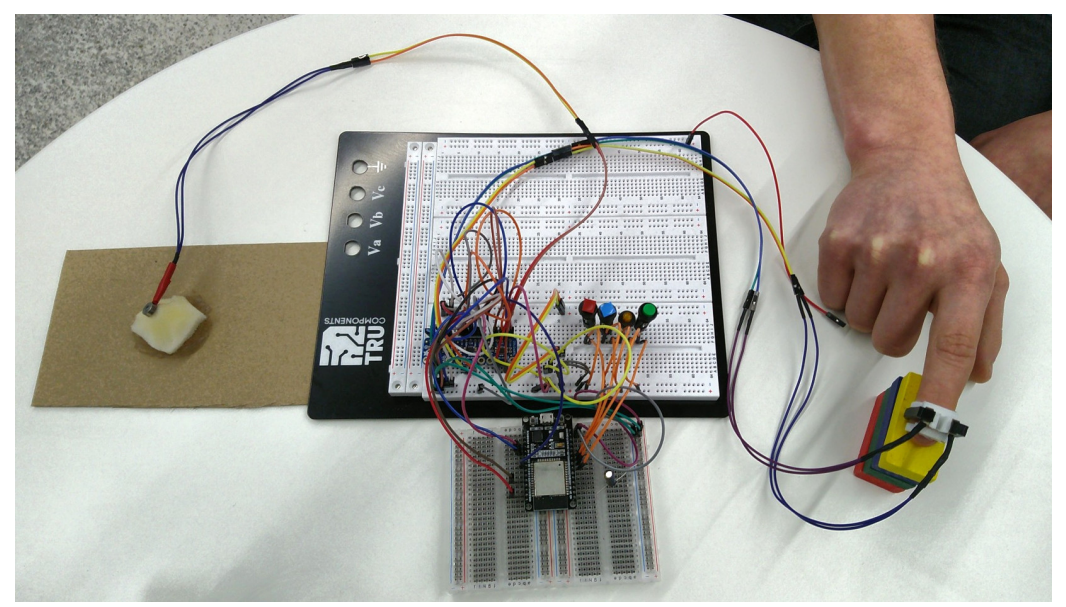

(a) Evaluation setup for the $2^{\text {nd }}$ task
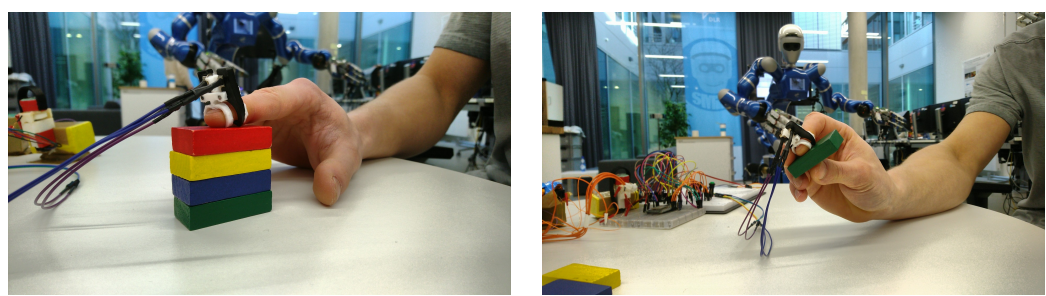

(b) Close-up views on the index finger illustrating the two subtasks on frequencies discrimination

Fig. 5. $2^{\text {nd }}$ task: discrimination of frequencies. Four different bricks representing four different vibration patterns were used during this subtask. The participants were blindfolded during the task and asked to find a brick with a specific color (respectively vibration pattern) by comparing it to the other bricks' vibration patterns (subtask 1), or to absolutely identify the color respectively pattern of a brick without being able to compare it to the other bricks (task 2).

Frequency detection subtask 2: For the second subtask, the experimenter handed the participant one brick and s/he had to identify the color. The participant was asked to rate the difficulty of identifying the color for all six repetitions. Each subject conducted both tasks subsequently.

Procedure: After a short introduction and signing informed consent, FingerTac was carefully placed on the first phalanx of the index finger of the dominant hand. Participants wore an ear protection to avoid them hearing the vibrations during all experiments. During the second task, the participants were blindfolded after the familiarization period. One experimenter wrote down the answers given by the participants.

Results: The $\mathrm{N}=10$ subjects detected the external system in $84.2 \%$ of the cases (significantly more often than guess probability, i.e. 50\%, $p<.001$ ), 
Table 2. Localization of vibrations

\begin{tabular}{|c|c|c|c|c|}
\hline & \multicolumn{2}{|c|}{ Detected } & Binomial Test \\
\hline & & "External Tactor" & "FingerTac" & Results \\
\hline \multirow{2}{*}{ Activated } & "External Tactor" & $84.2 \%$ & $15.8 \%$ & $p<.001$ \\
\hline & "FingerTac" & $46.2 \%$ & $53.8 \%$ & n.s. \\
\hline
\end{tabular}

while the detection rate for the functional demonstrator was $53.8 \%$, which is not significantly different from guess probability. This means that the subjects were not able to tell the origin of vibration if the functional demonstrator was active.

Frequency detection subtask 1: All frequencies were detected correctly in $100 \%$ of the cases. Accordingly, subjects indicated that it was rather easy to detect these frequencies with ratings ranging from $\mathrm{M}=1.6$ to $2.2(\mathrm{M}(1.38 \mathrm{~Hz})=$ $1.6 ; \mathrm{M}(3.63 \mathrm{~Hz})=2.1 ; \mathrm{M}(8 \mathrm{~Hz})=2.2 ; \mathrm{M}(13.33 \mathrm{~Hz})=1.6)$.

Frequency detection subtask 2: We found detection rates above $90 \%$ for the both lower frequencies $(92.3 \%$ for $1.38 \mathrm{~Hz}$ and $100 \%$ for $3.63 \mathrm{~Hz}$ ) while detection rates for the both higher frequencies reached $83.3 \%(8 \mathrm{~Hz})$ and $85.7 \%(13.33 \mathrm{~Hz})$.

Table 3. Frequency detection subtask 2

\begin{tabular}{ccccccc}
\hline & & \multicolumn{4}{c}{ Detected } \\
& & green & blue & yellow & red \\
\hline \multirow{4}{*}{ Activated } & green & $1.38 \mathrm{~Hz}$ & $\mathbf{9 2 . 3 \%}$ & $7.7 \%$ &.. &.. \\
blue & $3.63 \mathrm{~Hz}$ &.. & $\mathbf{1 0 0 \%}$ &.. &.. \\
& yellow & $8.00 \mathrm{~Hz}$ &.. & $16.7 \%$ & $\mathbf{8 3 . 3 \%}$ &.. \\
& red & $13.33 \mathrm{~Hz}$ &.. &.. & $14.3 \%$ & $\mathbf{8 5 . 7 \%}$ \\
\hline
\end{tabular}

The interrelation between activated and detected frequency was analyzed with Goodman und Kruskal's Lambda and showed a significant relation $(\lambda=$ $.87 ; p<.001)$. Although the difficulty ratings were higher compared to Task 1 they all ranged below the scale mean of $\mathrm{M}=4(\mathrm{M}(1.38 \mathrm{~Hz})=2.1 ; \mathrm{M}(3.63 \mathrm{~Hz})=$ $2.9 ; \mathrm{M}(8 \mathrm{~Hz})=3.6 ; \mathrm{M}(13.33 \mathrm{~Hz})=3.3)$.

\section{Conclusion}

This paper introduced FingerTac, which is a novel concept for a wearable tactile device for mobile augmented haptic applications. Its uniqueness is that it combines, for the first time, augmented haptics on a wearable light-weight device. As a result, it makes possible simultaneous tactile interaction with virtual and real objects.

A set of two evaluation studies was conducted to evaluate the effectiveness of the approach. It was observed that vibration transmission over a mechanical link is expedient when compared to a vibration actuator in direct contact with 
the skin, because it can result in better localized tactile feedback and slimmer device design. It was observed from the second study that by providing stimulus to the sides of a fingertip, the vibrations were felt in-between at the fingerpad. Furthermore, it could be verified that such device may be used to distinguish between various objects by providing different vibration patterns. In particular, a relative discrimination and assignment of four vibration patterns with different pause periods was easy with a detection rate of $100 \%$. An absolute mapping was still possible for more than $90 \%$ of all cases.

The next technological step is to design and build an integrated batterypowered version of the FingerTac device. This version may also contain enhancements, particularly with regard to additional sensors. It is also up to future work to investigate the relevance of the device for different mobile haptics applications. Since finger usage is task-dependent, using more than two fingers may be required for certain tasks. Thus, it would be essential for the user to get tactile feedback displayed on several fingers, which can be achieved using several FingerTac devices simultaneously.

\section{References}

1. Culbertson, H., Nunez, C.M., Israr, A., Lau, F., Abnousi, F., Okamura, A.M.: A social haptic device to create continuous lateral motion using sequential normal indentation. In: IEEE Haptics Symp. pp. 32-39 (2018)

2. De Rossi, D., Carpi, F., Carbonaro, N., Tognetti, A., Scilingo, E.P.: Electroactive polymer patches for wearable haptic interfaces. In: Int. Conf. of the IEEE Engineering in Medicine and Biology Society. pp. 8369-8372 (2011)

3. van Erp, J.B., van Veen, H.A.: A multi-purpose tactile vest for astronauts in the International Space Station. In: EuroHaptics. pp. 405-408. Dublin, Ireland: ACM, Press (2003)

4. Fani, S., Ciotti, S., Battaglia, E., Moscatelli, A., Bianchi, M.: W-FYD: A wearable fabric-based display for haptic multi-cue delivery and tactile augmented reality. IEEE Trans. on Haptics 11(2), 304-316 (2017)

5. Gabardi, M., Solazzi, M., Leonardis, D., Frisoli, A.: A new wearable fingertip haptic interface for the rendering of virtual shapes and surface features. In: IEEE Haptics Symp. pp. 140-146 (2016)

6. Gemperle, F., Ota, N., Siewiorek, D.: Design of a wearable tactile display. In: IEEE Int. Symp. on Wearable Computers. pp. 5-12 (2001)

7. Inoue, S., Makino, Y., Shinoda, H.: Active touch perception produced by airborne ultrasonic haptic hologram. In: IEEE World Haptics Conference (WHC). pp. 362 $367(2015)$

8. Jones, L.A., Sarter, N.B.: Tactile displays: Guidance for their design and application. Human factors 50(1), 90-111 (2008)

9. Komurasaki, S., Kajimoto, H., Ishizuka, H.: Fundamental perceptual characterization of an integrated tactile display with electrovibration and electrical stimuli. Micromachines 10(5), 301 (2019)

10. Leonardis, D., Solazzi, M., Bortone, I., Frisoli, A.: A 3-rsr haptic wearable device for rendering fingertip contact forces. IEEE Trans. on Haptics 10(3), 305-316 (2016)

11. Long, B., Seah, S.A., Carter, T., Subramanian, S.: Rendering volumetric haptic shapes in mid-air using ultrasound. ACM Trans. on Graphics (TOG) 33(6), 1-10 (2014) 
12. Minamizawa, K., Fukamachi, S., Kajimoto, H., Kawakami, N., Tachi, S.: Gravity grabber: wearable haptic display to present virtual mass sensation. In: ACM SIGGRAPH Computer Graphics (2007)

13. Moy, G., Wagner, C., Fearing, R.S.: A compliant tactile display for teletaction. In: IEEE Int. Conf. on Robotics and Automation (ICRA). vol. 4, pp. 3409-3415 (2000)

14. Mun, S., Yun, S., Nam, S., Park, S.K., Park, S., Park, B.J., Lim, J.M., Kyung, K.U.: Electro-active polymer based soft tactile interface for wearable devices. IEEE Trans. on Haptics 11(1), 15-21 (2018)

15. Nara, T., Takasaki, M., Tachi, S., Higuchi, T.: An application of saw to a tactile display in virtual reality. In: IEEE Ultrasonics Symp. vol. 1, pp. 1-4 (2000)

16. Pacchierotti, C., Salvietti, G., Hussain, I., Meli, L., Prattichizzo, D.: The hRing: A wearable haptic device to avoid occlusions in hand tracking. In: IEEE Haptics Symp. pp. 134-139. IEEE (2016)

17. Prattichizzo, D., Chinello, F., Pacchierotti, C., Malvezzi, M.: Towards wearability in fingertip haptics: a 3-dof wearable device for cutaneous force feedback. IEEE Trans. on Haptics 6(4), 506-516 (2013)

18. Schätzle, S., Ende, T., Wuesthoff, T., Preusche, C.: VibroTac: an ergonomic and versatile usable vibrotactile feedback device. In: IEEE Int. Symp. in Robot and Human Interactive Communication (Ro-Man). pp. 705-710. Viareggio, Italy (2010)

19. Schätzle, S., Hulin, T., Pleintinger, B.: VibroTac S: An electronic assistive device for blind and visually impaired people to avoid collisions. In: Int. Conf. on Human Systems Engineering and Design (IHSED). pp. 613-619. Springer (2018)

20. Scheibe, R., Moehring, M., Froehlich, B.: Tactile feedback at the finger tips for improved direct interaction in immersive environments. In: IEEE Symp. on 3D User Interfaces (3DUI) (2007)

21. Schorr, S.B., Okamura, A.M.: Fingertip tactile devices for virtual object manipulation and exploration. In: CHI Conf. on Human Factors in Computing Systems. pp. 3115-3119 (2017)

22. Singh, H., Suthar, B., Mehdi, S.Z., Ryu, J.H.: Ferro-fluid based portable fingertip haptic display and its preliminary experimental evaluation. In: IEEE Haptics Symp. pp. 14-19 (2018)

23. Solazzi, M., Frisoli, A., Bergamasco, M.: Design of a cutaneous fingertip display for improving haptic exploration of virtual objects. In: Int. Symp. in Robot and Human Interactive Communication. pp. 1-6. IEEE (2010)

24. Spelmezan, D., González, R.M., Subramanian, S.: Skinhaptics: Ultrasound focused in the hand creates tactile sensations. In: IEEE Haptics Symp. pp. 98-105 (2016)

25. Tsukada, K., Yasumura, M.: Activebelt: Belt-type wearable tactile display for directional navigation. In: Int. Conf. on Ubiquitous Computing. pp. 384-399. Springer (2004)

26. Velázquez, R., Pissaloux, E.E., Wiertlewski, M.: A compact tactile display for the blind with shape memory alloys. In: IEEE Int. Conf. on Robotics and Automation (ICRA). pp. 3905-3910 (2006)

27. Yang, G.H., Kyung, K.U., Srinivasan, M.A., Kwon, D.S.: Quantitative tactile display device with pin-array type tactile feedback and thermal feedback. In: IEEE Int. Conf. on Robotics and Automation (ICRA). pp. 3917-3922 (2006)

28. Yang, T.H., Kim, S.Y., Kim, C.H., Kwon, D.S., Book, W.J.: Development of a miniature pin-array tactile module using elastic and electromagnetic force for mobile devices. In: IEEE World Haptics Conference (WHC). pp. 13-17 (2009) 